Published in final edited form as:

J Health Care Poor Underserved. 2020 ; 31(4): 1669-1692. doi:10.1353/hpu.2020.0126.

\title{
Ideal Cardiovascular Health in Racially and Ethnically Diverse People with Serious Mental IIIness
}

\author{
Mark R. Hawes, \\ George Warren Brown School of Social Work at Washington University in St. Louis \\ Kimberly B. Roth, \\ Department of Community Medicine, Mercer University School of Medicine \\ Xiaoyan Wang, \\ George Warren Brown School of Social Work at Washington University in St. Louis \\ Ana Stefancic, \\ Department of Psychiatry at Columbia University \\ Christopher Weatherly, \\ George Warren Brown School of Social Work at Washington University in St. Louis \\ Leopoldo J. Cabassa \\ George Warren Brown School of Social Work at Washington University in St. Louis
}

\begin{abstract}
Cardiovascular disease is a primary contributor to premature death among people with serious mental illness (SMI). This study used baseline data $(\mathrm{N}=314)$ from an effectiveness trial testing a healthy lifestyle intervention for racial/ethnically diverse participants with overweight/obesity and SMI living in supportive housing. We examined the prevalence and correlates of a modified version of the American Heart Association (AHA) metric of ideal cardiovascular health (ICVH). Five AHA ICVH metrics (smoking, body mass index, diet, physical activity, and blood pressure) were used to create a composite ICVH score. The mean ICVH score was 3.15 (range $0-8$ ). Multivariate analysis indicated that higher ICVH scores were associated with lifetime cancer diagnosis and better cardiorespiratory fitness. Lower scores were associated with female gender, racial/ethnic minority status, and antipsychotic use suggesting that these subgroups may benefit from targeted screening and interventions to improve cardiovascular health in people with SMI.
\end{abstract}

\section{Keywords}

Serious mental illness; ideal cardiovascular health; cardiovascular disease; health disparities; racial and ethnic minority health

Cardiovascular disease (CVD) is a leading cause of morbidity and mortality in the United States and globally. ${ }^{1}$ Serious mental illness (SMI; e.g., schizophrenia, bipolar disorder)

Please address all correspondence to: Leopoldo J. Cabassa, George Warren Brown School of Social Work at Washington University in St. Louis, Goldfarb Hall, Campus Box 1196, One Brookings Drive, St. Louis, MO 63130; 1jcabassa@ wustl.edu. 
increases the risk of death from CVD by as much as $84 \% .^{2}$ People with SMI have a higher prevalence of CVD risk factors ${ }^{3}$ and mortality rates two to three times higher than the general population. ${ }^{4-7}$

In 2010, the American Heart Association (AHA) developed the concept of ideal cardiovascular health $(\mathrm{ICVH}),{ }^{8}$ a summative measure based on seven metrics that include four health behaviors (smoking, body mass index [BMI, $\mathrm{kg} / \mathrm{m} 2]$, diet, and physical activity [PA]) and three health indicators (cholesterol level, fasting glucose level, and blood pressure [BP]). Each metric is categorized into poor, intermediate, or ideal levels and assigned a score of 0,1 , or 2 , respectively. ${ }^{8,9}$ The points from each metric are summed to create a composite ICVH score where higher scores indicate better CVH. In contrast to the Framingham Risk Functions which estimate risk of developing CVD, ICVH does not focus on predicting risk, but rather on bringing a positive approach to prevention by focusing on health promotion. The ICVH metrics provide lifestyle goals and targets for clinicians to recommend and patients to achieve. ${ }^{9}$ For instance, one study integrated the ICVH metric into an electronic health record (EHR) as an assessment and intervention tool that providers could use with patients to monitor patients' cardiovascular health and help improve communication and shared decision making around $\mathrm{CVH} .{ }^{10}$

There have been numerous studies of ICVH in the general population and it is established that each ICVH metric is independently associated with overall CVH. ${ }^{1}$ Meeting a higher number of ICVH metrics is associated with lower risk for cancer, stroke, dementia, allcause, and CVD mortality, as well as a lower prevalence of depression, cognitive impairment, and higher self-rated health and quality of life. ${ }^{1-15}$ Achieving ICVH is rare in the U.S. population. Only $13 \%$ of the US population meets ideal levels on five metrics, $5 \%$ meet ideal levels on six metrics, and virtually none meet ideal levels across all seven metrics. ${ }^{1}$ Among adults in the general population, the metric with the highest proportion of people meeting ideal status is smoking (78.8\%); the metric with the lowest proportion of people meeting ideal status is diet $(<1 \%) .{ }^{1}$ Mirroring racial/ethnic disparities in CVD prevalence, risk behaviors, and risk factors, ${ }^{16-18} \mathrm{ICVH}$ also varies by race/ethnicity in the U.S. The prevalence of having ideal levels on four or more metrics is most common for Asians (48\%), followed by non-Hispanic Whites (38\%), Hispanics (34\%), Blacks (30\%), and other races $(24 \%){ }^{1}$

While ICVH has been studied in the general population, ${ }^{1,15}$ there is a dearth of studies examining the prevalence and correlates of ICVH among SMI populations, even though people with SMI consistently have elevated levels of CVD risk behaviors ${ }^{19-23}$ and risk factors. ${ }^{24}$ There is mixed evidence regarding racial/ethnic disparities in CVD risk factors among people with SMI. Some studies report no differences between Black and White people with SMI in most CVD risk factors, except for blood pressure, ${ }^{3}$ while other studies of SMI populations report higher risk for obesity in non-Hispanic Blacks and Hispanics compared with non-Hispanic Whites. ${ }^{25}$ We know of only one study (from Spain) that examined ICVH in 142 patients with SMI. ${ }^{26}$ They found three-quarters of their sample had suboptimal ICVH scores; most had ideal physical activity levels (84\%), whereas less than half had ideal BMI (16.9\%), diet (10.4\%), and smoking (47.9\%) status. 
Our study aimed to examine the prevalence of five ICVH metrics in a racially/ethnically diverse sample of people with SMI in supportive housing, and identify demographic, mental health, and physical health correlates of an adapted ICVH composite metric. Because our study used baseline data from an effectiveness trial testing a healthy lifestyle intervention for people with SMI and overweight/obesity, all participants had a BMI $\geq 25$ placing this sample at higher risk for poorer ICVH. Studying ICVH among this population of people with SMI living in the community is important since up to half of people with SMI have obesity ${ }^{27}$ and it will help fill the gap in what is known about CVH in racial and ethnic minorities with SMI. Examining ICVH will also provide an opportunity to improve the health of this population by informing future interventions and providing a composite metric, which includes modifiable risk factors (e.g., diet, physical activity) to track progress in improving CVH. Minor improvements in CVH can produce substantial reductions in CVD risk and death, and improvements in diet and physical activity levels are likely to yield some of the greatest benefits to $\mathrm{CVH}$ due to the substantial influence of these metrics on other $\mathrm{CVH}$ factors. ${ }^{15}$ There are no other studies to our knowledge examining the concept of ICVH in a racially/ethnically diverse sample of people with SMI in the U.S.

\section{Methods}

\section{Overview and study design.}

Our study used baseline data from a hybrid type 1 trial testing the effectiveness and examining the implementation of a peer-led group lifestyle balance (PGLB) program, a healthy lifestyle intervention, for participants with SMI in three supportive housing agencies. ${ }^{28}$ This trial is registered in clinicaltrials.gov (NCT02175641) and a detailed description of the study can be found in the PGLB study protocol. ${ }^{28}$ Briefly, participants were recruited through fliers, word-of-mouth, and staff referrals between 2015 and 2018. Research assistants conducted face-to-face interviews with interested participants to assess eligibility. Eligibility criteria included a chart diagnosis of a SMI, BMI of $\geq 25,18$ years of age or older, and the ability to provide informed consent and to obtain medical clearance. The sample for the current study includes all 314 participants who enrolled in the PGLB trial. The institutional review boards at both Columbia University and the Philadelphia Department of Health approved all study procedures.

\section{Measures.}

Ideal CVH metric.-Our ICVH metrics consisted of four health behaviors (smoking, BMI, $\mathrm{PA}$, and diet) and one health indicator (BP). The other two health indicators commonly used in the ICVH composite metric (cholesterol level, fasting glucose level) were not collected in the present study. Our measures for the five ICVH metrics included in this study closely correspond to the AHA recommendations, although, similar to other studies of ICVH, ${ }^{29-31}$ we adapted definitions for smoking and healthy diet components based on available data. Our ICVH metric definitions are described in Table 1. Each individual ICVH metric was scored as poor (0), intermediate (1), or ideal (2). Smoking status was self-reported. Participants who were not currently smoking were assigned an intermediate smoking status rather than ideal since the AHA definition of ideal requires that people do not smoke for more than one year and the PGLB study did not collect data on when former smokers quit. 
Research assistants measured participants' weight using a digital scale, and height using an anthropometric tape. Body mass index $(\mathrm{kg} / \mathrm{m} 2)$ was calculated from the participants' height and weight. Since participants were required to have a BMI $\geq 25$ in the PGLB trial, no participants had ideal BMI status. Physical activity (PA) was assessed by the International Physical Activity Questionnaire-Short Form (IPAQ), a self-report measure that captures participants' levels of PA. ${ }^{32}$ Fruit, vegetable, and fiber intake were measured using the Block fruit, vegetable, and dietary fat screeners. ${ }^{33}$ Sugar-sweetened beverage (SSB) intake was measured using questions from the 2013 Centers for Disease Control and Prevention Behavioral Risk Factors Surveillance System Questionnaire (BRFSS). ${ }^{34}$ Blood pressure (BP) was assessed on the right arm of participants after they rested quietly in a seated position for at least five minutes; the mean of two measurements was used for analysis. Based in part on the scoring system proposed by the developers of the ICVH construct, ${ }^{9,35}$ scores from each ICVH metric were summed to create a composite ICVH score that ranged from 0 to 8, with higher scores indicating better CVH. Since our study had five of the seven AHA metrics, we did not categorize the composite score into ideal, intermediate, and poor, but rather used the total score as our dependent variable.

\section{Correlates.}

Our selection of correlates was informed by findings from systematic literature reviews examining ICVH in the general population and studies examining correlates of CVD in people with SMI. ${ }^{1-3,15,25,26}$ All measures came from the baseline structured interview following the trial's measurement protocol. ${ }^{28}$ Demographic correlates were self-reported age, years of education, gender, racial/ethnic minority status, and employment status. Mental health correlates included self-reported lifetime physician-confirmed SMI diagnosis, including depression, schizophrenia/schizoaffective disorder, bipolar disorder, and substance abuse/dependence. Antipsychotic medication use was stratified into three categories: not taking an antipsychotic at the time of the baseline interview, taking only a first-generation antipsychotic (e.g., haloperidol, perphenazine), and taking a second generation (e.g., risperidone, quetiapine) or both second and first-generation antipsychotic. Health correlates included self-reported lifetime physician-confirmed diagnosis of high cholesterol, diabetes, CVD, and cancer. Consistent with other published studies, ${ }^{36,37}$ our CVD variable was defined as anyone who had a self-reported physician-confirmed diagnosis of coronary heart disease, stroke, arteriosclerosis, heart attack, or congestive heart failure. Number of health conditions was created by summing all medical conditions discussed above. Cardiorespiratory fitness (CRF; i.e., "the ability of the circulatory and respiratory systems to supply oxygen to working muscles during sustained physical activity" [p. 132] ${ }^{38}$ ) was assessed with the six-minute walking test (6MWT). The 6MWT measured the distance participants walked (in meters) at a normal pace along a flat and straight course for six minutes. ${ }^{28}$ Scores from the multidimensional internal health locus of control scale were also included. ${ }^{39}$

\section{Data analysis.}

All analyses were performed in Stata 16.0 (StataCorp LP, College Station, TX, USA). We described our overall sample based on demographic, health, and ICVH characteristics. Bivariate analysis was used to explore the relationships between our correlates and each 
ICVH metric and the composite ICVH score. Continuous variables were compared using ttests, Pearson correlation, or corresponding nonparametric tests based on distributional properties. Categorical variables were compared using chi-square or Fisher exact test. Hierarchal regression analysis was used to examine the associations of sample correlates with the total ICVH score. Correlates that were significant $(\mathrm{p}<.05)$ with any ICVH metric in the bivariate analysis were included in the multivariate model. A three-stage hierarchal multiple regression was conducted with the ICVH composite score as the dependent variable. The demographic block was entered at stage one, mental health block at stage two, and the physical health block at stage three. Changes in $\mathrm{R}^{2}$ were evaluated to test the contribution and significance of each block to the final model. Multiple linear regression assumptions of normality were assessed to confirm no violations of normality. The BreuschPagan and Cook-Weisberg tests found no problems with heteroskedasticity. We examined the variance inflation factor (VIF) for all independent variables; no variables had a VIF greater than 2 , indicating no multicollinearity issues. ${ }^{40}$ Outliers and influential data were identified using Cook's distance with the technical cutoff of $4 / n$. Seventeen outliers with values exceeding the cutoff were detected. Sensitivity analysis was conducted by excluding these 17 outliers from our models. There were no differences in model results, so the results for the full sample were reported. The final model had $7 \%$ missing data due to listwise deletion procedures.

\section{Results}

\section{Participant characteristics.}

Participant characteristics are presented in Table 2. The mean participant age was 48.65 years and average years of education was 11.91. More than half were male (57.32\%) and most were members of racial/ethnic minority groups (81.21\%), particularly non-Hispanic Blacks (57.64\%). The most commonly reported lifetime mental health diagnoses were depression (75.16\%), schizophrenia/schizoaffective disorder (56.69\%), and bipolar disorder $(46.50 \%)$. Approximately one-third (38.54\%) of participants reported a lifetime substance use/dependence problem. The majority (62.74\%) of participants were taking an antipsychotic: second-generation or combination of first- and second-generation (50\%), first-generation only (12.74\%). Participants' lifetime physical health diagnoses were high cholesterol (36.31\%), diabetes (32.48\%), CVD (17.20\%), and cancer (4.46\%). Participants walked an average of 318.42 meters during the 6MWT.

\section{Ideal CVH characteristics.}

Table 2 also displays ICVH characteristics. More than half of participants were current smokers $(62.74 \%)$ and had obesity (64.01\%). Most participants had poor (35.99\%) or intermediate (26.43\%) PA status. The majority of participants were categorized as having intermediate $(49.04 \%)$ or poor $(48.73 \%)$ dietary health and had either intermediate (38.39\%) or poor (38.06\%) BP status. On average, participants received 3.15 out of 8 possible points on the total ICVH score. 


\section{Bivariate analyses of ICVH correlates.}

We examined the relationship between each ICVH metric and our correlates (Table 3).

Being a current smoker was associated with older age $(\mathrm{p}=.048)$, less years of education $(\mathrm{p}<.001)$, female gender $(\mathrm{p}=.015)$, and lifetime substance abuse/dependence $(\mathrm{p}=.001)$.

Poor BMI status (obese) was associated with being a member of a racial/ethnic minority group $(\mathrm{p}=.021)$, high cholesterol $(\mathrm{p}=.010)$, lifetime diagnosis of diabetes $(\mathrm{p}=.008)$, and worse CRF ( $p<.001)$. There was a significant association between BMI status and lifetime cancer diagnosis $(\mathrm{p}=.023)$. A lower proportion of participants who reported a lifetime cancer diagnosis had obesity (35.71\%) compared with those without a cancer diagnosis $(65.55 \%)$.

Poor physical activity status was associated with having a lifetime diagnosis of schizophrenia $(\mathrm{p}=.005)$ and use of antipsychotic medications $(\mathrm{p}<.001)$. Ideal PA status was associated with having a lifetime diagnosis of depression $(\mathrm{p}=.025)$.

There was a significant association between gender and healthy diet status $(\mathrm{p}=.006)$ with a higher proportion of females than of males having poor diet status. The healthy diet metric was also significantly associated with diabetes $(\mathrm{p}=.024)$. Participants who reported having diabetes tended to have higher rates of intermediate diet status and lower rates of poor diet status.

Poor blood pressure status was significantly associated with older age ( $\mathrm{p}=.012)$ and racial/ ethnic minority status ( $\mathrm{p}=.002)$. A higher proportion of racial/ethnic minority group members had poor blood pressure status compared with non-Hispanic Whites.

The ICVH composite score was significantly associated with gender $(\mathrm{p}<.001)$ and racial/ ethnic minority status $(\mathrm{p}=.018)$, with females and racial/ethnic minority group members having lower mean scores. There was a statistically significant difference in the ICVH scores of participants taking a first-generation, second-generation, or no antipsychotic medication $(\mathrm{p}=.006)$. A Tukey post hoc test revealed that participants taking a first-generation antipsychotic had statistically significantly $(\mathrm{p}=.008)$ lower ICVH scores than participants not taking an antipsychotic. There was no statistically significant difference between participants who took second-generation versus only first-generation antipsychotics, or between participants taking second-generation antipsychotics and those not taking antipsychotics.

\section{Multivariate analysis.}

A three-stage hierarchal regression (Table 4) was conducted with the ICVH score as the dependent variable. In the first model, the demographic block contributed significantly to the regression model, $(F(4,298)=5.737, \mathrm{p}<.001)$ and explained $7.2 \%$ of the variance in ICVH scores. Racial/ethnic minority status and gender were the only demographic indicators independently associated with ICVH. The mental health block explained an additional 3.5\% of ICVH score variance. The change in $\mathrm{R}^{2}$ was significant $(F(9,289)=3.833, \mathrm{p}<.001)$. Gender and racial/ethnic minority status remained independent predictors as well as the use of antipsychotic medications. The addition of the physical health block explained an 
additional $4.2 \%$ of the variance in ICVH scores. The change in $\mathrm{R}^{2}$ was significant $(F(13$, $278)=3.731, \mathrm{p}<.001)$. In this final model, the following factors where significantly related to total ICVH: gender, racial/ethnic minority status, first- and second-generation antipsychotic use, cancer diagnosis, and CRF. Being female was associated with a 0.55 lower ICVH score $(\mathrm{p}=.002)$ and minority status was associated with a 0.63 decrease in ICVH scores $(\mathrm{p}=.006)$. Taking only a first-generation antipsychotic was associated with a 0.89 decrease in ICVH scores and taking a second-generation antipsychotic was associated with a 0.51 decrease in ICVH scores, compared with not using antipsychotics. Having a lifetime cancer diagnosis was associated with a 1.05 increase in ICVH scores. For every 50 additional meters walked during the 6MWT participants' ICVH scores increased by 0.12 . The final model accounted for $14.86 \%$ of the variance in ICVH scores.

\section{Discussion}

This is the first study that we are aware examining ICVH in a racially/ethnically diverse sample of individuals with SMI in the U.S. The low proportion of participants meeting ideal status on each ICVH metric and the low overall ICVH score is troublesome yet consistent with the fact that CVD risk behaviors (e.g., smoking, obesity, unhealthy diet) and risk factors (e.g., hypertension) are all significantly elevated in people with SMI. ${ }^{19-24}$ Multivariate analysis indicated that higher ICVH scores were associated with lifetime cancer diagnosis and better cardiorespiratory fitness. Lower scores were associated with female gender, racial/ethnic minority status, and antipsychotic use suggesting that these subgroups may benefit from targeted screening and interventions to improve cardiovascular health in people with SMI.

Our finding that females had lower ICVH scores than men was unexpected since this has not been observed in prior studies in the general population ${ }^{1}$ or in people with SMI. ${ }^{26}$ However, there is evidence from prior studies of cardiac risk estimates in people with schizophrenia indicating that females have higher cardiac risk than males. ${ }^{41}$ This gender disparity in ICVH scores should be explored further in other SMI populations. Differences in ICVH scores among male and females in our study may be explained by the higher prevalence of current smokers and poor dietary health status among females in our sample. The fact that females in our sample had a higher prevalence of smoking than males suggest that societal-level protective effects seen in the general population may not extend to populations with SMI. ${ }^{42}$ This finding and the overall high prevalence of smoking in our sample (62\%) highlights the need for smoking cessation interventions to be offered to all people with SMI. To accomplish this, more community mental health centers will need to begin offering smoking cessation services since many of these providers still do not include cessation treatment in their array of services. ${ }^{43}$

While lower ICVH scores among females were also driven by a higher prevalence of poor dietary status compared to males, overall only $2.23 \%$ of all participants met criteria for ideal dietary status. This is lower than the $10.4 \%$ found in another study of ICVH in people with SMI, ${ }^{26}$ but consistent with findings from a systematic review showing eight of 15 U.S. general population studies reported ideal diet prevalence of $1 \%$ or less and the remaining studies reported prevalence of $10 \%$ or less. ${ }^{15}$ Low ideal diet prevalence in our study may be 
partially explained by the fact that participants lived in supportive housing, had incomes below the poverty line, and the majority (90\%) received Supplemental Nutrition Assistance Program (SNAP) benefits. ${ }^{44,45}$ Supportive housing is more likely to be located in neighborhoods with limited access to fresh fruits and vegetables. ${ }^{46}$ Further, living below the poverty line and receiving SNAP benefits may decrease access to resources that facilitate engagement in healthy dietary habits in people with SMI. ${ }^{46,47}$ The low prevalence of ideal diet in our sample is a matter for concern since dietary behaviors directly affect $\mathrm{CVH}^{15}$ and suggests that diet as well as access to healthful foods may be important intervention targets to improve the health of people with SMI.

The fact that members of racial/ethnic minority groups in our sample had significantly lower ICVH scores than non-Hispanic Whites is consistent with studies of the general population $^{1,51}$ that find similar racial/ethnic disparities in ICVH scores. However, few studies have examined these health disparities in ICVH in racial and ethnic minorities with SMI, particularly among people living in community settings. ${ }^{25}$ The differences in ICVH scores between non-Hispanic Whites and racial/ethnic minorities in our sample may be driven by the higher prevalence of obesity and poor BP among our minority participants, who were mostly non-Hispanic Black. Previous studies have reported similar disparities in obesity and poor BP among Blacks with SMI compared with non-Hispanic Whites with SMI. ${ }^{3,25}$ Our findings support the need for developing interventions to focus on improving ICVH in members of racial/ethnic minority groups with SMI, particularly those that address issues of obesity and high BP.

In our sample, those taking antipsychotics had lower ICVH scores than participants not taking antipsychotics. Further, people taking first-generation antipsychotics had lower ICVH scores than those taking second-generation antipsychotics. It is possible that antipsychotic use could be a proxy for illness severity which could affect engagement in unhealthy behaviors, thus negatively affecting CVH. It is known that antipsychotics induce weight gain $^{48}$ thus influencing participants' ICVH scores, but in our study all participants had a BMI $\geq 25$, and antipsychotic use was not associated with BMI status. Antipsychotic use is also associated with engaging in less $\mathrm{PA}^{23}$ and this was confirmed in our sample as well. Sixty percent of participants were taking an antipsychotic, and only $37 \%$ of participants in our study achieved ideal PA status, which is less than the $50 \%$ of people with SMI reported to meet physical activity guidelines in two metanalysis. ${ }^{20,23}$ Lower levels of PA among antipsychotic users in our study would have resulted in lower ICVH scores for these participants.

Lifetime cancer diagnosis was associated with higher ICVH composite scores. A plausible explanation for this finding is that these participants could be connected to medical care due to their cancer diagnosis, which could explain their higher ICVH scores. Since cancer was reported as a lifetime diagnosis, we could not ascertain when participants had cancer. More work in this area is needed to examine the relationship between cancer and ICVH in people with SMI.

Our finding that participants with better CRF had higher ICVH scores, even while controlling for covariates, is consistent with the findings of Bueno-Antequera. ${ }^{26}$ Poor CRF is 
known to be a significant predictor of all-cause mortality ${ }^{49}$ and is significantly lower in people with SMI when compared with the general population. ${ }^{50}$ Research indicates that CRF can be improved with increases in $\mathrm{PA}^{50}$ and there are interventions, such as In Shape, ${ }^{51}$ that have shown promise in improving PA and CRF in people with SMI. Taken together, these findings indicate that future interventions should target improvements in CRF among people with SMI.

\section{Limitations.}

The modified ICVH metric used in this study was missing two ICVH health factors (cholesterol level, fasting glucose level) recommended by the AHA. Future studies in people with SMI should include all ICVH indicators. Despite this limitation, studying the four behavioral metrics and one health indicator metric available in this study provides an important contribution to the literature. According to the 2019 AHA statistical update report, each ICVH metric is independently associated with overall $\mathrm{CVH},{ }^{1}$ and diet and PA, examined in this study, are the two metrics likely to yield the greatest improvements in CVH due to their influence on other ICVH metrics. ${ }^{15}$ The clinical difference between individuals categorized as having ideal, intermediate, or poor $\mathrm{CVH}$ may be small and this increases the risk of misclassification which could be further exacerbated by self-report data. Self-report measures were used to evaluate levels of PA, diet, smoking, and many of our correlates; these measures tend to over- or under-estimate the prevalence of variables and are subject to recall-bias. ${ }^{52,53}$ While self-report measures are imperfect, they are commonly used in large community studies due to their feasibility and are used extensively in population-based studies for examination and surveillance of health. Future studies should try to use objective measures to reduce these biases and generate more precise estimates of ICVH and of the relationships between ICVH and correlates. The AHA categorizes individuals as having ideal smoking status if they never smoked or if they quit for greater than one year. ${ }^{8}$ Nonsmokers in our study could not be categorized as ideal since the original study did not collect data on when participants quit smoking. Our data were drawn from a sample of participants living in three supportive housing agencies participating in an effectiveness trial, which limits the generalizability of our findings to this service setting. However, supportive housing is an important service setting for people with SMI since it provides (along with community housing) physical health, mental health, and social services. Since the majority of our racial/ethnic minority population was non-Hispanic Black and Hispanic, we analyzed racial/ethnic minorities in aggregate. Given the heterogeneity of racial/ethnic minorities, studies are needed that disaggregate racial and ethnic group data and carefully examine within and between group differences to develop a more nuanced understanding of ICVH in these diverse populations. The participants in this study all had a BMI $\geq 25$, which limits generalizability, although given that up to $55 \%{ }^{27}$ of people with SMI have obesity, this is an important subpopulation that should be closely examined. Our correlates focused on individual-level factors. Future studies should examine multilevel factors to understand better the macro-level forces (e.g., built environment) that influence the $\mathrm{CVH}$ of people with SMI. For instance, future studies should examine how access to healthful foods and opportunities for physical activity affect ICVH. Lastly, our cross-sectional analysis prevented us from making casual inferences. Longitudinal studies are needed to examine the pathways that influence ICVH in people with SMI. 


\section{Conclusion.}

In our sample of people with SMI in supportive housing, females, racial and ethnic minorities, and those taking antipsychotic medications have lower ICVH scores, indicating that these may be important subgroups that could benefit from targeted screening and health interventions. Our finding that members of racial/ethnic minority groups fared worse than non-Hispanic Whites adds to the limited knowledge of CVH in minority group members with SMI. Moreover, the association between CRF and ICVH scores in our sample highlights the importance of increasing PA and reducing sedentary behavior in people with SMI since both are modifiable risk factors that can improve CVH.

In order to promote health, given the strong evidence that each of the ICVH metrics is associated with overall $\mathrm{CVH}$, it is important that clinicians begin making routine the assessment of each of the ICVH metrics. As has been done in the general population, ${ }^{10}$ community mental health centers could use the ICVH metrics to create EHR tools to assess and intervene on overall CVH. Routinely assessing the ICVH metrics will allow for work to be done that evaluates the association of ICVH with a multitude of factors that affect the health and quality of life of people with SMI. Our study demonstrates how the ICVH metric can be a useful tool to understand the CVH of people with SMI. As national and global efforts to improve the physical health of people with SMI continue to grow, ICVH can be used as an important population-based metric to track the progress of these efforts and help identify areas to improve the CVH of people with SMI.

\section{Acknowledgments}

Research reported in this publication was supported by the National Institute of Mental Health (NIMH) under award number (R01MH104574 and P50MH115843). Authors MH and CW are supported as pre-doctoral fellows and author KR as a post-doctoral fellow by NIMH award number (T32MH019960). The content is solely the responsibility of the authors and does not necessarily represent the official views of the National Institutes of Health. The authors would like to thank the study participants and community partners for their engagement in this study.

\section{References}

1. Benjamin EJ, Muntner P, Alonso A, et al. Heart Disease and Stroke Statistics-2019 Update: A Report From the American Heart Association. Circulation. 2019;139(10):56-528. doi:10.1161/ CIR.0000000000000659

2. Correll CU, Solmi M, Veronese N, et al. Prevalence, incidence and mortality from cardiovascular disease in patients with pooled and specific severe mental illness: a large-scale meta-analysis of 3,211,768 patients and 113,383,368 controls. World Psychiatry. 2017;16(2):163-180. doi:10.1002/ wps.20420 [PubMed: 28498599]

3. Keenan TE, Yu A, Cooper LA, et al. Racial patterns of cardiovascular disease risk factors in serious mental illness and the overall U.S. population. Schizophr Res. 2013;150(1):211-216. doi:10.1016/ j.schres.2013.07.022 [PubMed: 23916188]

4. Olfson M, Gerhard T, Huang C, Crystal S, Stroup TS. Premature mortality among adults with schizophrenia in the United States. JAMA Psychiatry. 2015;72(12):1172-1181. doi:10.1001/ jamapsychiatry.2015.1737 [PubMed: 26509694]

5. Hayes JF, Marston L, Walters K, King MB, Osborn DPJ. Mortality gap for people with bipolar disorder and schizophrenia: UK-based cohort study 2000-2014. Br J Psychiatry. 2017;211(3):175181. doi:10.1192/bjp.bp.117.202606 [PubMed: 28684403]

6. Colton CW, Manderscheid RW. Congruencies in increased mortality rates, years of potential life lost, and causes of death among public mental health clients in eight states. Prev Chronic Dis. 
2006;3(2):1-14. http://www.ncbi.nlm.nih.gov/pubmed/16539783\%0Ahttp://

www.pubmedcentral.nih.gov/articlerender.fcgi?artid=PMC1563985

7. Osborn DPJ, Levy G, Nazareth I, Petersen I, Islam A, King MB. Relative Risk of Cardiovascular and Cancer Mortality in People With Severe Mental Illness From the United Kingdom's General Practice Research Database. Arch Gen Psychiatry. 2007;64(2):242. doi:10.1001/archpsyc.64.2.242 [PubMed: 17283292]

8. Lloyd-Jones DM, Hong Y, Labarthe D, et al. Defining and Setting National Goals for Cardiovascular Health Promotion and Disease Reduction. Circulation. 2010;121(4):586-613. doi:10.1161/CIRCULATIONAHA.109.192703 [PubMed: 20089546]

9. Lloyd-Jones DM. Improving the Cardiovascular Health of the US Population. JAMA. 2012;307(12):1314. doi:10.1001/jama.2012.361 [PubMed: 22427616]

10. Foraker RE, Shoben AB, Lopetegui MA, et al. Assessment of Life's Simple 7TM in the primary care setting: The Stroke Prevention in Healthcare Delivery EnviRonmEnts (SPHERE) study. Contemp Clin Trials. 2014;38(2):182-189. doi:10.1016/j.cct.2014.03.007 [PubMed: 24721482]

11. Bergman E, Löyttyniemi E, Rautava P, Veromaa V, Korhonen PE. Ideal cardiovascular health and quality of life among Finnish municipal employees. Prev Med Reports. 2019;15(June):100922. doi:10.1016/j.pmedr.2019.100922

12. Folsom AR, Yatsuya H, Nettleton JA, Lutsey PL, Cushman M, Rosamond WD. Community Prevalence of Ideal Cardiovascular Health, by the American Heart Association Definition, and Relationship With Cardiovascular Disease Incidence. J Am Coll Cardiol. 2011;57(16):1690-1696. doi:10.1016/j.jacc.2010.11.041 [PubMed: 21492767]

13. Manczuk M, Vaidean G, Dehghan M, Vedanthan R, Boffetta P, Zatonski WA. Ideal cardiovascular health is associated with self-rated health status. The Polish Norwegian Study (PONS). Int J Cardiol. 2017;230:549-555. doi:10.1016/j.ijcard.2016.12.060 [PubMed: 28043659]

14. Sabia S, Fayosse A, Dumurgier J, et al. Association of ideal cardiovascular health at age 50 with incidence of dementia: 25 year follow-up of Whitehall II cohort study. BMJ. 2019;366:14414. doi:10.1136/bmj.14414 [PubMed: 31391187]

15. Younus A, Aneni EC, Spatz ES, et al. A Systematic Review of the Prevalence and Outcomes of Ideal Cardiovascular Health in US and Non-US Populations. Mayo Clin Proc. 2016;91(5):649670. doi:10.1016/j.mayocp.2016.01.019 [PubMed: 27040086]

16. Daviglus ML, Talavera GA, Avilés-Santa ML, et al. Prevalence of Major Cardiovascular Risk Factors and Cardiovascular Diseases Among Hispanic/Latino Individuals of Diverse Backgrounds in the United States. JAMA. 2012;308(17):1775. doi:10.1001/jama.2012.14517 [PubMed: 23117778]

17. Young DR, Fischer H, Arterburn D, et al. Associations of overweight/obesity and socioeconomic status with hypertension prevalence across racial and ethnic groups. J Clin Hypertens. 2018;20(3):532-540. doi:10.1111/jch.13217

18. Hales CM, Carroll MD, Fryar CD, Ogden CL. Prevalence of Obesity Among Adults and Youth: United States, 2015-2016. NCHS Data Brief, No 288; 2017.

19. Glasheen C, Hedden SL, Forman-Hoffman VL, Colpe LJ. Cigarette smoking behaviors among adults with serious mental illness in a nationally representative sample. Ann Epidemiol. 2014;24(10):776-780. doi:10.1016/j.annepidem.2014.07.009 [PubMed: 25169683]

20. Stubbs B, Firth J, Berry A, et al. How much physical activity do people with schizophrenia engage in? A systematic review, comparative meta-analysis and meta-regression. Schizophr Res. 2016;176(2-3):431-440. doi:10.1016/j.schres.2016.05.017 [PubMed: 27261419]

21. Allison DB, Newcomer JW, Dunn AL, et al. Obesity Among Those with Mental Disorders. Am J Prev Med. 2009;36(4):341-350. doi:10.1016/j.amepre.2008.11.020 [PubMed: 19285199]

22. Dipasquale S, Pariante CM, Dazzan P, Aguglia E, McGuire P, Mondelli V. The dietary pattern of patients with schizophrenia: A systematic review. J Psychiatr Res. 2013;47(2):197-207. doi:10.1016/j.jpsychires.2012.10.005 [PubMed: 23153955]

23. Vancampfort D, Firth J, Schuch FB, et al. Sedentary behavior and physical activity levels in people with schizophrenia, bipolar disorder and major depressive disorder: a global systematic review and meta-analysis. World Psychiatry. 2017;16(3):308-315. doi:10.1002/wps.20458 [PubMed: 28941119] 
24. Gardner-Sood P, Lally J, Smith S, et al. Cardiovascular risk factors and metabolic syndrome in people with established psychotic illnesses: Baseline data from the IMPaCT randomized controlled trial. Psychol Med. 2015;45(12):2619-2629. doi:10.1017/S0033291715000562 [PubMed: 25961431]

25. Carliner H, Collins PY, Cabassa LJ, McNallen A, Joestl SS, Lewis-Fernández R. Prevalence of cardiovascular risk factors among racial and ethnic minorities with schizophrenia spectrum and bipolar disorders: a critical literature review. Compr Psychiatry. 2014;55(2):233-247. doi:10.1016/ j.comppsych.2013.09.009 [PubMed: 24269193]

26. Bueno-Antequera J, Oviedo-Caro MÁ, Munguía-Izquierdo D. Ideal cardiovascular health and its association with sedentary behaviour and fitness in psychiatric patients. The PsychiActive project. Nutr Metab Cardiovasc Dis. 2018;28(9):900-908. doi:10.1016/j.numecd.2018.05.003 [PubMed: 29898823]

27. Janssen EM, McGinty EE, Azrin ST, Juliano-Bult D, Daumit GL. Review of the evidence: prevalence of medical conditions in the United States population with serious mental illness. Gen Hosp Psychiatry. 2015;37(3):199-222. doi:10.1016/j.genhosppsych.2015.03.004 [PubMed: 25881768]

28. Cabassa LJ, Stefancic A, O'Hara K, et al. Peer-led healthy lifestyle program in supportive housing: Study protocol for a randomized controlled trial. Trials. 2015;16(1):1-14. 10.1186/ s13063-015-0902-z [PubMed: 25971836]

29. Bambs C, Kip KE, Dinga A, Mulukutla SR, Aiyer AN, Reis SE. Low Prevalence of "Ideal Cardiovascular Health" in a Community-Based Population. Circulation. 2011;123(8):850-857. doi:10.1161/CIRCULATIONAHA.110.980151 [PubMed: 21321154]

30. Kim JI, Sillah A, Boucher JL, Sidebottom AC, Knickelbine T. Prevalence of the American Heart Association's "Ideal Cardiovascular Health" Metrics in a Rural, Cross-sectional, CommunityBased Study: The Heart of New Ulm Project. J Am Heart Assoc. 2013;2(3):1-11. doi:10.1161/ JAHA.113.000058

31. Simon M, Boutouyrie P, Narayanan K, et al. Sex disparities in ideal cardiovascular health. Heart. 2017;103(20):1595-1601. doi:10.1136/heartjnl-2017-311311 [PubMed: 28754808]

32. Craig CL, Marshall AL, Sjostrom M, et al. International Physical Activity Questionnaire: 12Country Reliability and Validity. Med Sci Sport Exerc. 2003;35(8):1381-1395. doi:10.1249/01.MSS.0000078924.61453.FB

33. Glady B, Christina G, Ernes TR, Christopher J. A rapid food screener to assess Fats and fruits and vegetables intake. Am J Prev Med. 2000;18(4):284-288. [PubMed: 10788730]

34. Centers for Disease Control and Prevention. Behavioral Risk Factor Surveillance System Questionnaire (BRFSS). Atlanta, Georgia.; 2013.

35. Huffman MD, Capewell S, Ning H, Shay CM, Ford ES, Lloyd-Jones DM. Cardiovascular Health Behavior and Health Factor Changes (1988-2008) and Projections to 2020. Circulation. 2012;125(21):2595-2602. doi:10.1161/CIRCULATIONAHA.111.070722 [PubMed: 22547667]

36. Cabassa LJ, Lewis-Fernández R, Wang S, Blanco C. Cardiovascular disease and psychiatric disorders among Latinos in the United States. Soc Psychiatry Psychiatr Epidemiol. 2017;52(7):837-846. doi:10.1007/s00127-016-1325-1 [PubMed: 28062923]

37. Goodwin RD, Davidson KW, Keyes K. Mental disorders and cardiovascular disease among adults in the United States. J Psychiatr Res. 2009;43(3):239-246. doi:10.1016/j.jpsychires.2008.05.006 [PubMed: 18614179]

38. Vancampfort D, Rosenbaum S, Probst M, et al. Promotion of cardiorespiratory fitness in schizophrenia: a clinical overview and meta-analysis. Acta Psychiatr Scand. 2015;132(2):131-143. doi:10.1111/acps.12407 [PubMed: 25740655]

39. Wallston KA. The Validity of the Multidimensional Health Locus of Control Scales. J Health Psychol. 2005;10(5):623-631. doi:10.1177/1359105305055304 [PubMed: 16033784]

40. Kutner MH, Nachtsheim C, \& Neter J Applied Linear Regression Models. McGraw-Hill/Irwin; 2007.

41. Goff DC, Sullivan LM, McEvoy JP, et al. A comparison of ten-year cardiac risk estimates in schizophrenia patients from the CATIE study and matched controls. Schizophr Res. 2005;80(1):45-53. [PubMed: 16198088] 
42. Hartz SM, Pato CN, Medeiros H, et al. Comorbidity of Severe Psychotic Disorders With Measures of Substance Use. JAMA Psychiatry. 2014;71(3):248. doi:10.1001/jamapsychiatry.2013.3726 [PubMed: 24382686]

43. Brunette MF, Ferron JC, McGurk SR, et al. Brief, Web-Based Interventions to Motivate Smokers With Schizophrenia: Randomized Trial. JMIR Ment Heal. 2020;7(2):e16524. doi:10.2196/16524

44. Cabassa LJ, Stefancic A, Lewis-Fernaández R, et al. (In Press). Main outcomes of the peer-led healthy lifestyle intervention for people with serious mental illness in supportive housing. Psychiatric Services.

45. Weinstein LC, Chilton M, Turchi R, et al. Reaching for a healthier lifestyle: a photovoice investigation of healthy living in people with serious mental illness. Prog Community Heal Partnerships Res Educ Action. 2019;13(4):371-83. 10.1353/cpr.2019.0061

46. Henwood BF, Cabassa LJ, Craig CM, Padgett DK. Permanent Supportive Housing: Addressing Homelessness and Health Disparities? Am J Public Health. 2013;103(S2):S188-S192. doi:10.2105/AJPH.2013.301490 [PubMed: 24148031]

47. Cabassa LJ, Parcesepe A, Nicasio A, Baxter E, Tsemberis S, Lewis-Fernández R. Health and Wellness Photovoice Project. Qual Health Res. 2013;23(5):618-630. doi:10.1177/1049732312470872 [PubMed: 23258117]

48. Tek C, Kucukgoncu S, Guloksuz S, Woods SW, Srihari VH, Annamalai A. Antipsychotic-induced weight gain in first-episode psychosis patients: a meta-analysis of differential effects of antipsychotic medications. Early Interv Psychiatry. 2016;10(3):193-202. doi:10.1111/eip.12251 [PubMed: 25962699]

49. Kodama S, Saito K, Tanaka S, et al. Cardiorespiratory Fitness as a Quantitative Predictor of AllCause Mortality and Cardiovascular Events in Healthy Men and Women. JAMA. 2009;301(19):2024-2035. doi:10.1001/jama.2009.681 [PubMed: 19454641]

50. Vancampfort D, Rosenbaum S, Schuch F, et al. Cardiorespiratory Fitness in Severe Mental Illness: A Systematic Review and Meta-analysis. Sport Med. 2017;47(2):343-352. doi:10.1007/ s40279-016-0574-1

51. Bartels SJ, Pratt SI, Aschbrenner KA, et al. Clinically significant improved fitness and weight loss among overweight persons with serious mental illness. Psychiatr Serv. 2013;64(8):729-736. doi:10.1176/appi.ps.003622012 [PubMed: 23677386]

52. Duncan MJ, Arbour-Nicitopoulos K, Subramanieapillai M, Remington G, Faulkner G. Revisiting the International Physical Activity Questionnaire (IPAQ): Assessing physical activity among individuals with schizophrenia. Schizophr Res. 2017;179:2-7. doi:10.1016/j.schres.2016.09.010 [PubMed: 27623360]

53. Lee PH, Macfarlane DJ, Lam T, Stewart SM. Validity of the international physical activity questionnaire short form (IPAQ-SF): A systematic review. Int J Behav Nutr Phys Act. 2011;8(1):115. doi:10.1186/1479-5868-8-115 [PubMed: 22018588] 
Table 1.

Ideal Cardiovascular Health (ICVH) Metrics and Definitions

\begin{tabular}{|c|c|c|}
\hline ICVH Metrics & Definitions & \\
\hline Smoking & - & $\begin{array}{l}\text { Poor: Current smoker } \\
\text { Intermediate: Not a current smoker }\end{array}$ \\
\hline Body mass index & - & $\begin{array}{l}\text { Poor: } 230 \mathrm{~kg} / \mathrm{m}^{2} \\
\text { Intermediate: } 25-29.9 \mathrm{~kg} / \mathrm{m}^{2}\end{array}$ \\
\hline Physical activity & $\begin{array}{l}\cdot \\
\cdot \\
\cdot\end{array}$ & $\begin{array}{l}\text { Poor: No min/wk of mod or vig } \\
\text { Intermediate: } 1-149 \mathrm{~min} / \mathrm{wk} \mathrm{mod}, 1-74 \mathrm{~min} / \mathrm{wk} \text { vig, or } 1-149 \mathrm{~min} / \mathrm{wk} \mathrm{mod}+\mathrm{vig} \\
\text { Ideal: } \geq 150 \mathrm{~min} / \mathrm{wk} \mathrm{mod} \text {, or } \geq 75 \mathrm{~min} / \mathrm{wk} \text { vig, or } \geq 150 \mathrm{~min} \mathrm{mod}+\mathrm{vig}\end{array}$ \\
\hline Healthy Diet Score & $\begin{array}{l}\cdot \\
\cdot \\
\cdot\end{array}$ & $\begin{array}{l}\text { Poor: } 0 \text { components } \\
\text { Intermediate: } 1-2 \text { components } \\
\text { Ideal: } 3 \text { components }\end{array}$ \\
\hline Blood Pressure & $\begin{array}{l} \\
\cdot \\
-\end{array}$ & $\begin{array}{l}\text { Poor: Systolic } \geq 140 \mathrm{~mm} \mathrm{Hg} \text { or diastolic } \geq 90 \mathrm{~mm} \mathrm{Hg} \\
\text { Intermediate: Systolic } 120-139 \mathrm{~mm} \mathrm{Hg} \text { / diastolic } 80-89 \mathrm{~mm} \mathrm{Hg} \\
\text { Ideal: Systolic }<120 \mathrm{~mm} \mathrm{Hg} \text { / diastolic }<80 \mathrm{~mm} \mathrm{Hg}\end{array}$ \\
\hline
\end{tabular}

Note: Ideal=2 Points; Intermediate=1 Point; Poor =0 Points.

Min = minutes $; \mathrm{wk}=$ week Mod = moderate physical activity Vig = vigorous physical activity

To define a healthy diet, we included three of the five American Heart Association (AHA) dietary components. Healthy diet score components included the following: $\geq 4.5$ cups of fruits and vegetables per day; 25 grams of fiber per day; $\leq 36$ oz of sugary sweetened beverages per week. Healthy diet status was categorized based on number of dietary components met: ideal ( 3 components), intermediate (1-2 components), poor ( 0 components). 
Table 2.

Baseline demographic and clinical characteristics of all participants enrolled $(\mathrm{N}=314)^{a}$

\begin{tabular}{|c|c|c|c|c|}
\hline & $\mathbf{N}$ & $\%$ & Mean & SD \\
\hline \multicolumn{5}{|l|}{ Demographics } \\
\hline Age at baseline & 313 & & 48.65 & 11.56 \\
\hline Total years of education & 310 & & 11.91 & 2.48 \\
\hline \multicolumn{5}{|l|}{ Gender } \\
\hline Male & 180 & 57.32 & & \\
\hline Female & 133 & 42.36 & & \\
\hline Other $b$ & 1 & 0.32 & & \\
\hline \multicolumn{5}{|l|}{ Race/ethnicity } \\
\hline Racial/ethnic minority & 255 & 81.21 & & \\
\hline Non-Hispanic Black & 181 & 57.64 & & \\
\hline Non-Hispanic White & 57 & 18.15 & & \\
\hline Hispanic & 39 & 12.42 & & \\
\hline Non-Hispanic Other & 35 & 11.15 & & \\
\hline \multicolumn{5}{|l|}{ Current employment status } \\
\hline Unemployed & 281 & 89.49 & & \\
\hline Employed & 32 & 10.19 & & \\
\hline \multicolumn{5}{|l|}{ Mental Health } \\
\hline \multicolumn{5}{|c|}{ Lifetime self-reported physician- confirmed diagnosis of psychiatric disorders ${ }^{c}$} \\
\hline Depression & 236 & 75.16 & & \\
\hline Schizophrenia / Schizoaffective disorder & 178 & 56.69 & & \\
\hline Bipolar disorder & 146 & 46.50 & & \\
\hline Substance abuse / dependence & 121 & 38.54 & & \\
\hline \multicolumn{5}{|l|}{ Antipsychotic medication $^{d}$} \\
\hline Not taking an antipsychotic & 117 & 37.26 & & \\
\hline $1^{\text {st }}$ Generation & 40 & 12.74 & & \\
\hline $2^{\text {nd }}$ Generation or combination of $2^{\text {nd }}$ and $1^{\text {st }}$ Generation & 157 & 50.00 & & \\
\hline \multicolumn{5}{|l|}{ Physical Health } \\
\hline \multicolumn{5}{|c|}{ Lifetime self-reported physician-confirmed diagnosis of chronic medical conditions ${ }^{c}$} \\
\hline High cholesterol & 114 & 36.31 & & \\
\hline Diabetes & 102 & 32.48 & & \\
\hline Cardiovascular disease $^{e}$ & 54 & 17.20 & & \\
\hline Cancer & 14 & 4.46 & & \\
\hline Number of physical health conditions & 314 & & 3.66 & 2.41 \\
\hline \multicolumn{5}{|l|}{ Cardiorespiratory fitness } \\
\hline 6-minute walk-test (meters) & 313 & & 318.42 & 96.87 \\
\hline \multicolumn{5}{|l|}{ Health Agency } \\
\hline Internal health locus of control ${ }^{f}$ & 314 & & 28.68 & 4.77 \\
\hline
\end{tabular}




\begin{tabular}{|c|c|c|c|c|}
\hline & $\mathbf{N}$ & $\%$ & Mean & SD \\
\hline \multicolumn{5}{|l|}{ Ideal Cardiovascular Health (ICVH) Metrics } \\
\hline \multicolumn{5}{|l|}{ Smoking status } \\
\hline Intermediate (Not currently smoking) & 117 & 37.26 & & \\
\hline Poor (Current smoker) & 197 & 62.74 & & \\
\hline \multicolumn{5}{|l|}{$\mathrm{BMI}^{g}$ status, $\mathrm{kg} / \mathrm{m} 2$} \\
\hline Intermediate $(25-29.9 \mathrm{~kg} / \mathrm{m} 2)$ & 113 & 35.99 & & \\
\hline Poor ( $\geq 30 \mathrm{~kg} / \mathrm{m} 2)$ & 201 & 64.01 & & \\
\hline \multicolumn{5}{|l|}{ Physical activity status ${ }^{h}$} \\
\hline Ideal ( $2150 \mathrm{~min} / \mathrm{wk}$ of moderate or $75 \mathrm{~min} / \mathrm{wk}$ vigorous; or combination of 150 or greater) & 118 & 37.58 & & \\
\hline Intermediate ( 1 to $149 \mathrm{~min} / \mathrm{wk}$ or $-74 \mathrm{~min} / \mathrm{wk}$ vigorous, or combination of $-149 \mathrm{~min} / \mathrm{wk}$ ) & 83 & 26.43 & & \\
\hline Poor $(0 \mathrm{~min} / \mathrm{wk})$ & 113 & 35.99 & & \\
\hline \multicolumn{5}{|l|}{ Healthy diet status } \\
\hline Ideal (3 components) & 7 & 2.23 & & \\
\hline Intermediate ( 1 to 2 components) & 154 & 49.04 & & \\
\hline Poor ( 0 components) & 153 & 48.73 & & \\
\hline \multicolumn{5}{|l|}{ Blood pressure status } \\
\hline Ideal $(\mathrm{SBP}<120 / \mathrm{DBP}<80 \mathrm{~mm} \mathrm{Hg})$ & 73 & 23.55 & & \\
\hline Intermediate (SBP 120-139/DBP 80-89 mm Hg) & 119 & 38.39 & & \\
\hline Poor (SBP $\geq 140$ or DBP $\geq 0 \mathrm{~mm} \mathrm{Hg}$ ) & 118 & 38.06 & & \\
\hline Total score $(0-8)^{i}$ & 310 & & 3.15 & 1.49 \\
\hline
\end{tabular}

${ }^{a}$ Percentages of participant characteristics do not always add up to $100 \%$ due to missing data for some variables.

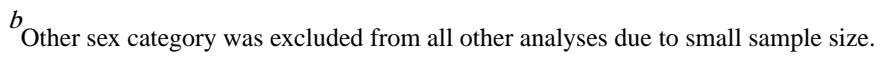

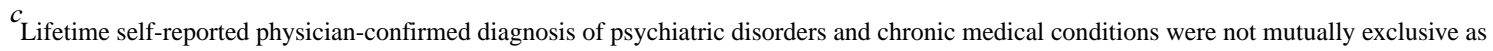
participants could have more than one condition, therefore percentages add up to greater than 100 .

$d_{1}$ st generation includes participants taking only first generation/typical antipsychotics; $2^{\text {nd }}$ generation includes participants taking only second generation/atypical antipsychotics and those taking both a first- and second-generation antipsychotic.

${ }^{e}$ Cardiovascular disease was defined as anyone who had a self-reported physician-confirmed diagnosis of coronary artery disease, stroke, arteriosclerosis, heart attack, or congestive heart failure.

Internal health locus of control score ranges from 6 to 36, with higher numbers indicating stronger belief in internal health locus of control.

$g_{\text {Body mass index. }}$

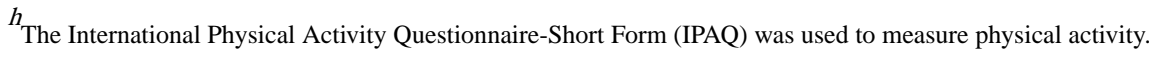

${ }^{i}$ Scores from each ICVH metric were summed to create a composite ICVH score that ranges from 0 to 8 , with higher scores indicating better cardiovascular health. ICVH total scores were computed for 310 out of 314 participants because four participants were missing blood pressure status. 


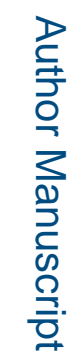

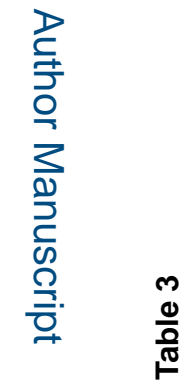

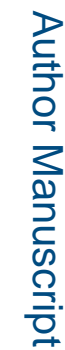

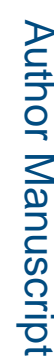

J Health Care Poor Underserved. Author manuscript; available in PMC 2021 January 09. 


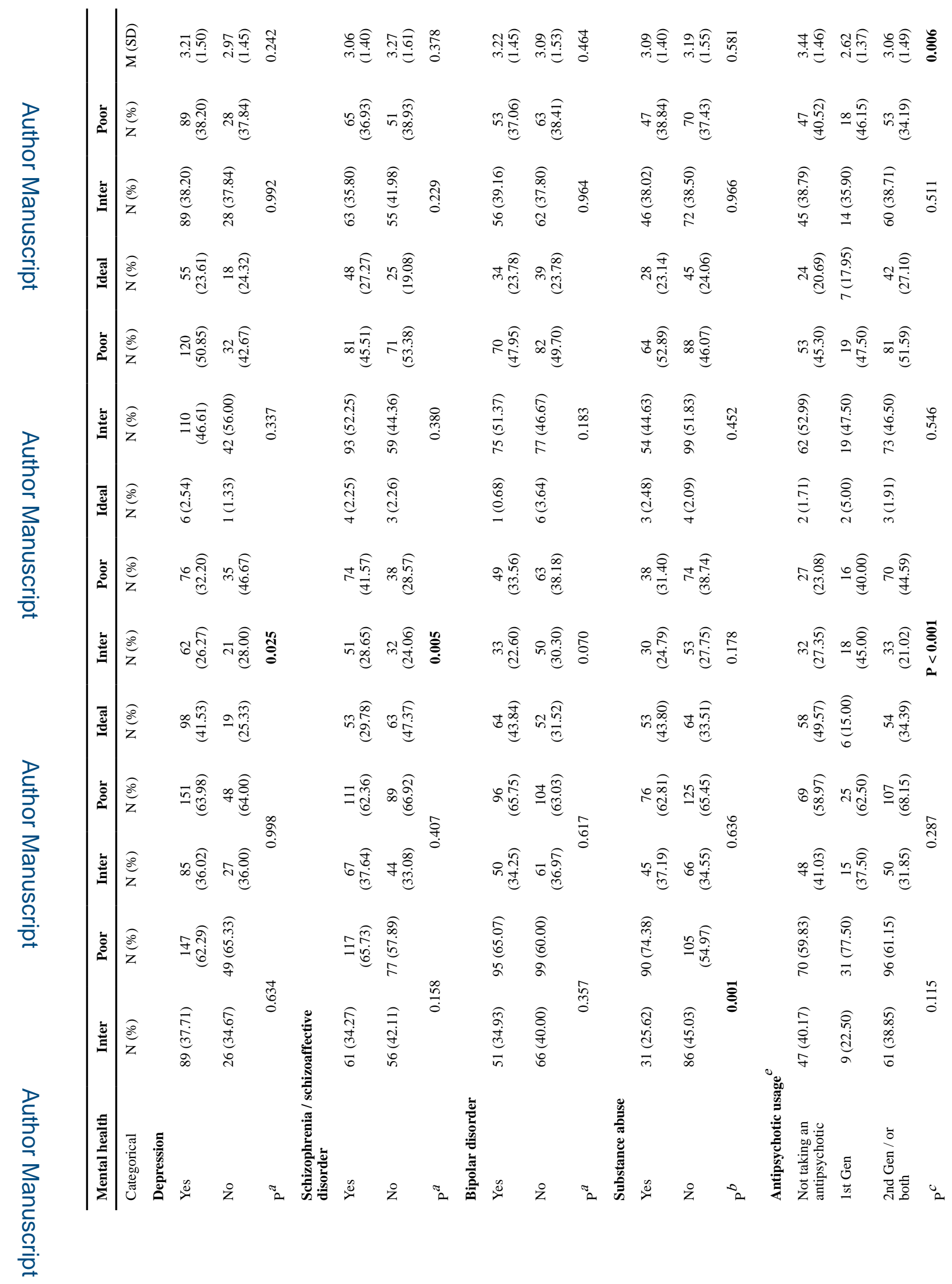

J Health Care Poor Underserved. Author manuscript; available in PMC 2021 January 09. 


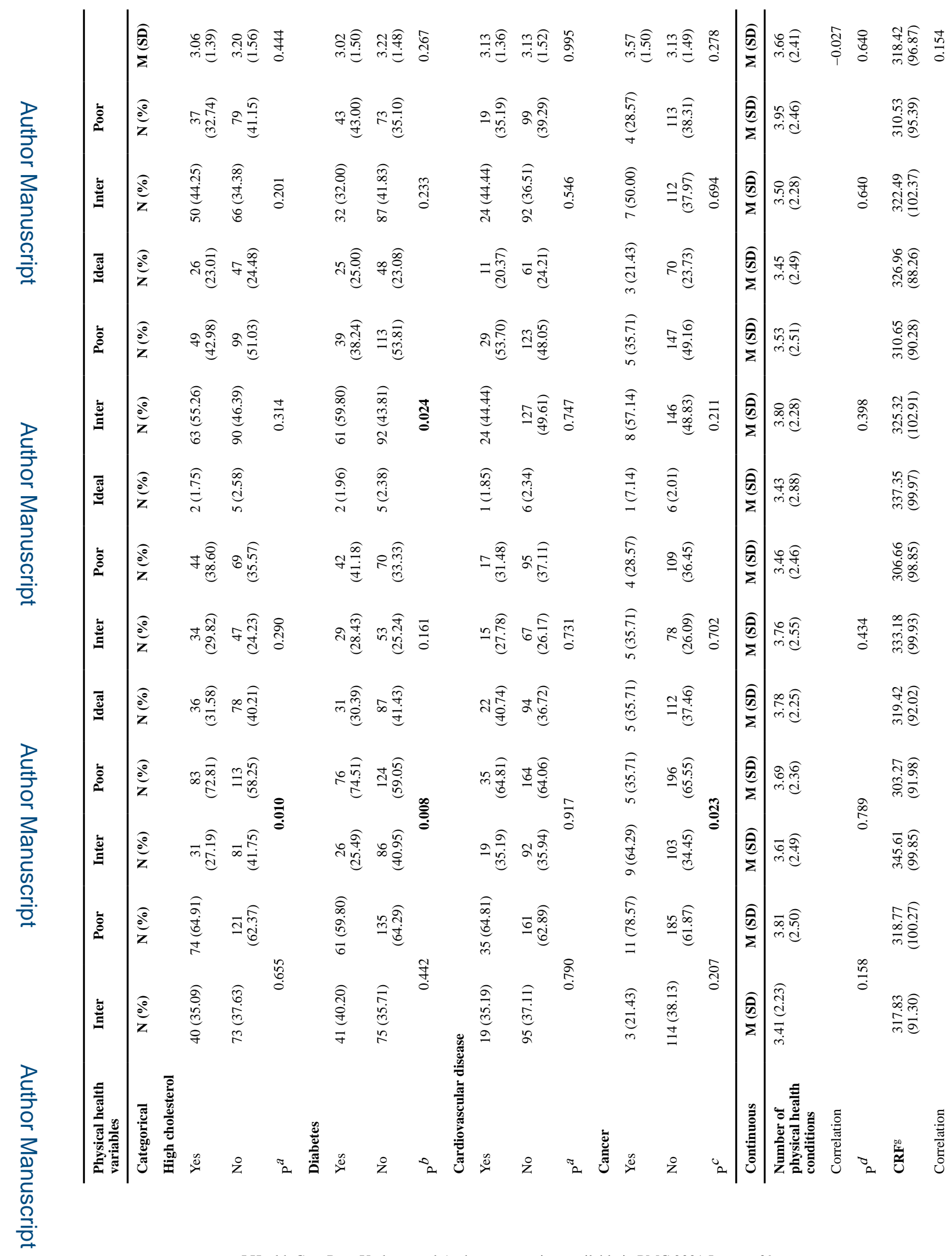

J Health Care Poor Underserved. Author manuscript; available in PMC 2021 January 09. 
Hawes et al.

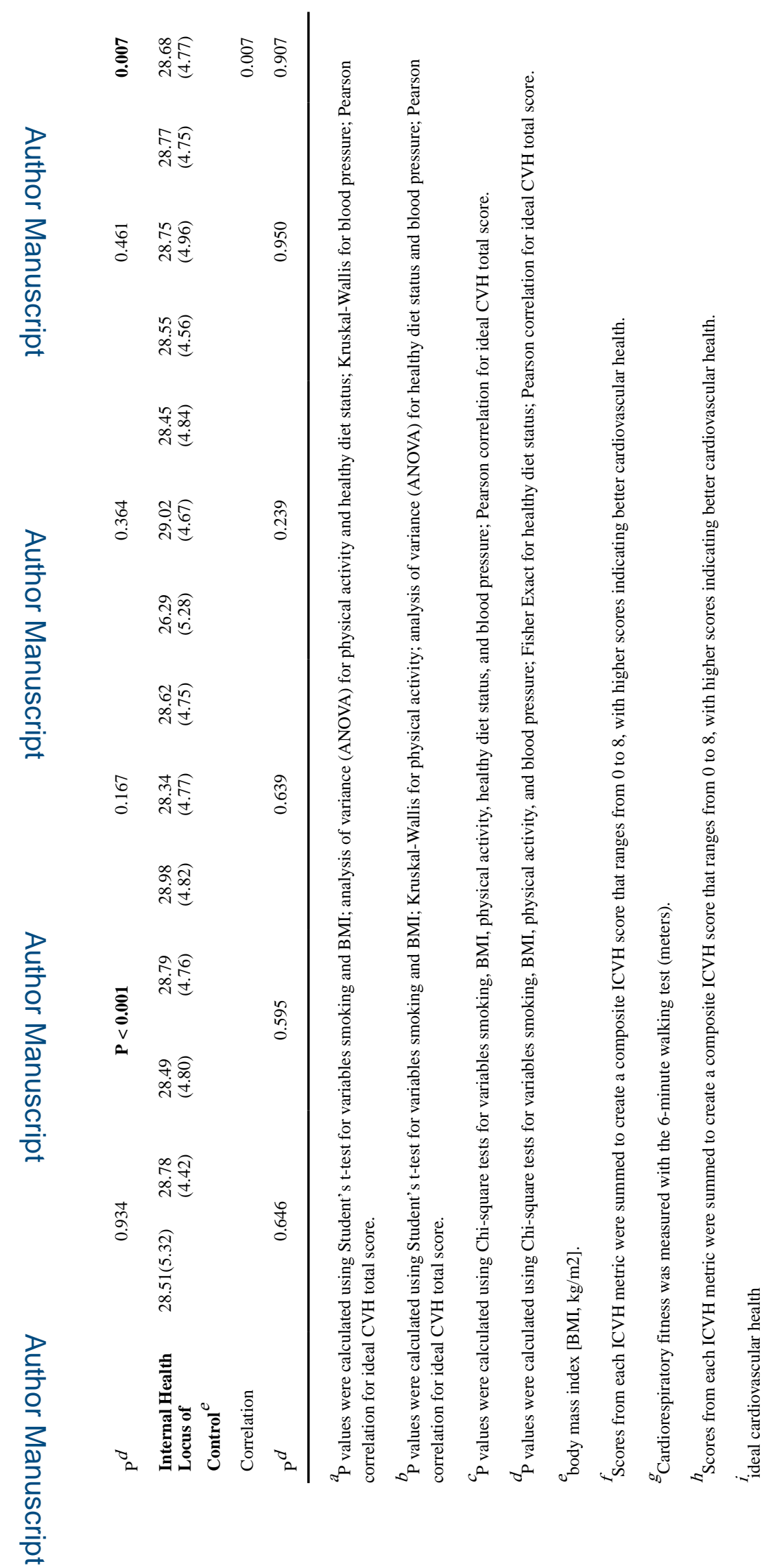

J Health Care Poor Underserved. Author manuscript; available in PMC 2021 January 09. 


\section{Table 4}

Regression analysis of ideal cardiovascular health score

\begin{tabular}{|c|c|c|c|c|c|c|c|c|c|}
\hline & \multicolumn{3}{|c|}{ Model 1} & \multicolumn{3}{|c|}{ Model 2} & \multicolumn{3}{|c|}{ Model 3} \\
\hline & Coef. $^{a}$ & SE & $\mathbf{P}$ & Coef. $^{a}$ & SE & $\mathbf{P}$ & Coef. $^{a}$ & SE & $\mathbf{P}$ \\
\hline \multicolumn{10}{|l|}{ Demographics } \\
\hline Age at baseline & -0.14 & 0.07 & 0.067 & -0.13 & 0.07 & 0.074 & -0.11 & 0.08 & 0.1766 \\
\hline Years of education & 0.03 & 0.03 & 0.141 & 0.05 & 0.04 & 0.182 & 0.06 & 0.04 & 0.097 \\
\hline Female (Ref: Male) & -0.61 & 0.17 & $\mathbf{P}<0.001$ & -0.60 & 0.17 & $\mathbf{P}<0.001$ & -0.55 & 0.17 & 0.002 \\
\hline Racial/ethnic minority (Ref: Non-Hispanic white) & -0.52 & 0.22 & 0.017 & -0.54 & 0.22 & 0.014 & -0.63 & 0.23 & 0.006 \\
\hline \multicolumn{10}{|l|}{ Mental Health } \\
\hline Depression (Ref: No) & & & & 0.26 & 0.21 & 0.219 & 0.30 & 0.22 & 0.163 \\
\hline Schizophrenia/Schizoaffective Disorder (Ref: No) & & & & 0.11 & 0.19 & 0.571 & 0.17 & 0.19 & 0.390 \\
\hline Substance Abuse or Dependence (Ref: No) & & & & -0.20 & 0.18 & 0.270 & -0.30 & 0.18 & 0.090 \\
\hline 1st Generation Antipsychotic (Ref: No) & & & & -0.76 & 0.31 & 0.014 & -0.89 & 0.31 & 0.004 \\
\hline 2nd Generation Antipsychotic (Ref: No) $b$ & & & & -0.42 & 0.20 & 0.033 & -0.51 & 0.20 & 0.011 \\
\hline \multicolumn{10}{|l|}{ Physical Health } \\
\hline High Cholesterol (Ref: No) & & & & & & & -0.09 & 0.19 & 0.622 \\
\hline Diabetes (Ref: No) & & & & & & & 0.15 & 0.21 & 0.473 \\
\hline Cancer (Ref: No) & & & & & & & 1.05 & 0.45 & 0.019 \\
\hline CRF (6MWT, 50m increase) ${ }^{c}$ & & & & & & & 0.12 & 0.04 & 0.009 \\
\hline Analytic N & & & 303 & & & 299 & & & 292 \\
\hline $\mathrm{R}^{2}$ & & & .072 & & & 0.107 & & & 149 \\
\hline $\mathrm{R}^{2}$ change & & & & & & $.035^{*}$ & & & $042^{*}$ \\
\hline F Statistic & & & $.74^{* *}$ & & & $83^{* * *}$ & & & $73^{* *}$ \\
\hline \multicolumn{10}{|c|}{ a Notes: Calculated using ordinary least squares regression. } \\
\hline \multicolumn{10}{|c|}{$\begin{array}{l}b_{2} \text { nd } \text { generation includes participants taking only second generation/atypical antipsychotics and those taking both a first- and second-generation } \\
\text { antipsychotic. }\end{array}$} \\
\hline \multicolumn{10}{|l|}{ 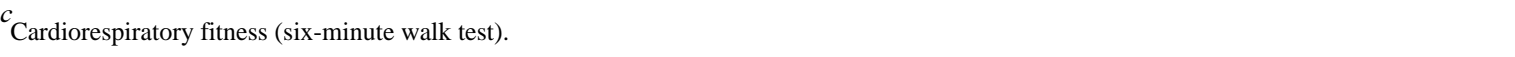 } \\
\hline \multicolumn{10}{|l|}{${ }^{*}<0.05$} \\
\hline * ${ }^{*}<0.001$ & & & & & & & & & \\
\hline
\end{tabular}

